\title{
EDITORIAL
}

\section{MILIEU MARIN, HYDROLOGIE ET PHARMACIE}

A maintes reprises participant à des réunions consacrées à la mer, j'ai pu constater un certain étonnement chez mes interlocuteurs apprenant ma qualité d'hydrologue et de pharmacien.

Les questions posées me reviennent à l'esprit au moment où j'écrit ces lignes, à bord du navire océanographique NOROIT, en mission d'êtude biologique en Méditerranée entre la SARDAIGNE et les BALEARES.

Certes la situation semble assez surprenante au premier abord, mais pour de multiples raisons que je voudrais évoquer ici, il me parait tout-à-fait logique que l'hydrologue et le pharmacien s'intéressent au milieu marin.

Au cours des siècles la tradition a établi, entre le domaine continental et l'océan, une distinction aussi tranchée que celle existant entre le terrien et le marin. Cet état de fait s'est concrétisé par l'organisation d’administrations, de règlementations différentes et même, dans une large mesure, par des recherches distinctes.

Certes, chacun de ces domaines possède ses caractéristiques propres, mais n'est-il pas exagéré d'agir comme si une barrière infranchissable les séparait. 
En fait, les milieux aquatiques ne sont pas constitués de blocs indépendants, sans influence les uns sur les autres.

Si I'on admet facilement l'interdépendance des diffée rentes composantes des eaux continentales, on ne peut plus limiter leurs relations avec la mer aux seuls estuaires ou à quelques étangs littoraux, et ignorer les conséquences de leur dispersion lointaine dans le domaine marin. Il est impossible à I'heure actuelle de traiter des eaux douces comme si elles ne devaient jamais quitter le continent et il devient impératif d'envisager tous problèmes de gestion et de qualité les concernant en tenant compte des caractéristiques propres du milieu océanique qui, tôt ou tard, les recevra.

L'impact sur le littoral est évidemment le plus spectaculaire car cette zone est, en définitive, assez confinée à cause de son découpage, de son relief et des courants particuliers qui l'isolent souvent du large.

Ainsi l'arrivée des eaux continentales se fait d'abord dans un domaine de faible capacité dont la masse d'eau réduite et les fonds rassemblent la plus grande partie de la richesse biologique de la mer. Cette frange est la première à subir les conséquences de la qualité des déversements naturels et artificiels, sans compter les effets néfastes et, quelquefois, les ravages provoqués par l'aménagement de la côte et des cours d'eau (barrages - détournements) qui rompent l'equilibre fragile entre les eaux douces et les eaux salées.

Mais tous les effets des activités continentales ne sont pas limités à la zone littorale ; ils se prolongent beaucoup plus loin par deux voies bien distinctes : l'eau et l'air.

Ainsi les grands fleuves entrainent vers le large des eaux dont l'assimilation n'interviendra que très lentement et ils apportent surtout une charge solide macro et microscopique fortement imprégnée de la pollution acquise dans tous leur bassin versant.

Je ne citerai pour preuve que les tristes déchets récupérés par nos chaluts à 3000 mètres de profondeur à quelques 300 ou 400 kilomè tres de nos côtes méditerranéennes, et à la présence de quantités notables de polluants dans les eaux interstitielles des vases sur lesquelles ils étaient déposés.

Mais la relation entre les domaines continentaux et océaniques n'est pas seulement limitée aux mélanges de leurs eaux, et les rapports qui s'établissent entre-eux par la voie aérienne prennent à notre époque une importance de plus en plus grande. 
Ainsi, les embruns ramènent vers la terre une solution chargée en polluants domestiques ou industriels particulièrement nocifs non seulement pour la flore mais également pour les populations littorales. De tels retours sont particulièrement préoccupants en mer du Nord qui est largement utilisée comme zone de rejets de résidus industriels provenant de divers pays de la communauté europénne.

Dans l'autre sens, le transport sur de grandes distances de tout le matériel particulaire émis au-dessus des zones industrielles atteint en grande partie les océans et constitue d'après certains une part essentielle de la pollution des mers.

En présence de tels échanges, on peut se demander s'il est souhaitable de maintenir le cloisonnement que nous citions ci-dessus aussi bien dans l'administration que dans la recherche concernant chacun de ces domaines.

Ne serait-il pas souhaitable d'arriver à une conception plus générale des milieux aquatiques conduisant à une sciences pluridisciplinaire seule capable d'élucider les problèmes actuels de l'eau.

La part que peut prendre le pharmacien dans cette étude et cette défence du milieu marin, et plus particulièrement des zones littorales, ne doit pas être sous-estimée. Si sa vocation d'hygiéniste, de microbiologiste, d'analyste et de toxicologue lui permet d'apporter une contribution non négligeable à la résolution de multiples problèmes de pollution, je soulignerai en particulier l'apport qui peut être le sien dans certains domaines comme la molysmocinétique dont la démarche est très voisine de celle de la pharmacocinétique.

Mais au-delà de ces aspects généraux liés à la qualité du milieu marin, il importe au pharmacien de s.intéresser à un domaine qui peut, professionnellement, lui apporter beaucoup.

Nous envisagions tout-à-l'heure la richesse biologique de la mer dont on a un peu trop tendance à ne considérer que le côté alimentaire, certes essentiel, alors que la thçrapeutique a tant à en attendre. On peut dire, sans être grand prophète qu'un avenir riche de promesses s'ouvre à la recherche et à l'étude de produits nouveaux que la biologie marine nous offre. II serait impensable que la faune et la flore continentales nous aient tant apporté et que la mer dont nous sommes issus nous fournisse seulement de I'huile de foie de morue et de l'iode.

Dans un ordre d'idée quelque peu différent, il serait illogique à une époque où l'on redécouvre les vertus de la crénothérapie, de négliger la thalassothérapie qui ne doit pas sombrer dans les erreurs passées du thermalisme. 
Si la variété chimique des eaux marines ne présente pas la diversité des eaux minérales, il est tout aussi évident qu'une meilleure connaissance des propriétés biologiques et de l'utilisation des diverses composantes du milieu marin devrait permettre, en s'appuyant sur une véritable recherche médicale, d'ouvrir quelques horizons sur autre chose qu'une thérapeutique à la mode chez quelques privilégiés ou initiés.

André ARNOUX 